This is the author's final, peer-reviewed manuscript as accepted for publication. The publisher-formatted version may be available through the publisher's web site or your institution's library.

\title{
Biochemical characterization of chitin synthase activity and inhibition in the African malaria mosquito, Anopheles gambiae
}

Xin Zhang and Kun Yan Zhu

\section{How to cite this manuscript}

If you make reference to this version of the manuscript, use the following information:

Zhang, X., \& Zhu, K. Y. (2013). Biochemical characterization of chitin synthase activity and inhibition in the African malaria mosquito, Anopheles gambiae. Retrieved from http://krex.ksu.edu

\section{Published Version Information}

Citation: Zhang, X., \& Zhu, K. Y. (2013). Biochemical characterization of chitin synthase activity and inhibition in the African malaria mosquito, Anopheles gambiae. Insect Science, 20(2), 158-166.

Copyright: (c) 2012 Institute of Zoology, Chinese Academy of Sciences

Digital Object Identifier (DOI): doi:10.1111/j.1744-7917.2012.01568.x

Publisher's Link:

http://onlinelibrary.wiley.com/doi/10.1111/j.1744-7917.2012.01568.x/full

This item was retrieved from the K-State Research Exchange (K-REx), the institutional repository of Kansas State University. K-REx is available at http://krex.ksu.edu 
1 Submit to: Insect Science

MS\#: INS-2012-05-151

2 For special section: Insect Chitin Metabolism

3

4

5

6

7

8

9

10

21 Correspondence: Kun Yan Zhu, Department of Entomology, 123 Waters Hall, Kansas State

22 University, Manhattan, KS 66506, USA. Fax: 785-532-6232; Email: kzhu@ksu.edu 24 KS 66506, USA.

\section{African malaria mosquito, Anopheles gambiae}

\author{
Xin Zhang ${ }^{\dagger}$ and Kun Yan Zhu \\ Department of Entomology, Kansas State University, Manhattan, KS 66506, USA
}

Running Head: Mosquito chitin synthase activity and inhibition

17 University, Manhattan, KS 66506, USA. Fax. 785-532-6232; Email: kzhu@ksu.edu

${ }^{\dagger}$ Current address: Division of Biology, 116 Ackert Hall, Kansas State University, Manhattan, 


\section{Abstract}

26 Chitin synthase (CHS) is an important enzyme catalyzing the formation of chitin polymers in all

27 chitin containing organisms and a potential target site for insect pest control. However, our 28 understanding of biochemical properties of insect chitin synthases has been very limited. We

29 here report enzymatic and inhibitory properties of chitin synthase prepared from the African

30 malaria mosquito, Anopheles gambiae. Our study, which represents the first time to use a

31 nonradioactive method to assay chitin synthase activity in an insect species, determined the

32 optimal conditions for measuring the enzyme activity, including $\mathrm{pH}$, temperature, and

33 concentrations of the substrate UDP- $N$-acetyl-D-glucosamine (GlcNAc) and $\mathrm{Mg}^{++}$. The optimal

$34 \mathrm{pH}$ was about 6.5-7.0, and the highest activity was detected at temperatures between 37 and

$3544^{\circ} \mathrm{C}$. Dithithreitol is required to prevent melanization of the enzyme extract. CHS activity was

36 enhanced at low concentration of GlcNAc, but inhibited at high concentrations. Proteolytic

37 activation of the activity is significant both in the $500 \mathrm{xg}$ supernatant and the 40,000xg pellet. Our

38 study revealed only slight in vitro inhibition of An. gambiae CHS activity by diflubenzuron and

39 nikkomycin $\mathrm{Z}$ at the highest concentration $(2.5 \mu \mathrm{M})$ examined. There was no in vitro inhibition

40 by polyoxin $\mathrm{D}$ at any concentration examined. Furthermore, we did not observe any in vivo

41 inhibition of chitin synthase activity by any of these chemicals at any concentration examined.

42 Our results suggest that the inhibition of chitin synthesis by these chemicals is not due to direct

43 inhibition of chitin synthase in An. gambiae.

46 Key words: Anophele gambiae, chitin synthase, diflubenzuron, enzyme inhibition 


\section{Introduction}

49 Chitin, a linear polysaccharide of $\mathrm{N}$-acetyl- $\beta$-D-glucosamine residues joined by $\beta-1,4$ glycosidic

50 linkages, is the second most abundant biological polymer after cellulose (Merzendorfer, 2006;

51 Kramer \& Muthukrishnan, 2005). It is widely distributed in arthropods, fungi, nematodes and

52 other Phyla such as annelids, molluscs and coelenterates. In arthropods, chitin is a vital

53 component of the cuticular exoskeleton and thus is crucial for growth and development

54 (Merzendorfer \& Zimoch, 2003). Chitin is also found in internal structures of many insects and

55 other arthropods, including the cuticular linings of trachea and in the peritrophic matrixes (PM)

56 lining the gut epithelium (Richards, 1951; Hunt, 1970; Cohen, 2001).

Chitin production in arthropods is a complicated process and a series of biochemical

58 pathways are involved in individual chitin polymer biosynthesis in which the terminal step is

59 catalyzed by chitin synthase (CHS, EC2.4.1.16), which is a large transmembrane protein that

60 belongs to the family of $\beta$-glycosyltransferases. CHS catalyzies the transfer of sugar moieties

61 from activated sugar donors to specific acceptors in all chitin-containing organisms. In insects,

62 chitin synthase contains multiple transmembrane helices reflecting their association with either

63 the plasma membrane or intracellular vesicles such as chitosomes (Tellam et al., 2000).

As insect and fungi growth and development depend on precisely tuned expression of

65 CHS (Arakane et al., 20005, 2008; Merzendorfer, 2006) and chitin is not synthesized by

66 vertebrates, CHS presents an attractive target for combating insect pests and fungi-born diseases

67 (Merzendorfer, 2006). For example, peptidyl nucleosides including polyoxins and nikkomycins

68 are anti-fungi agents which competitively inhibit CHS in fungi and insects (Cohen \& Casida,

69 1980b; Zhang \& Miller, 1999; Ruiz-Herrera \& San-Blas, 2003), whereas benzylphenolureas

70 (BPUs) such as diflubenzuron are highly effective insecticides which inhibit chitin synthesis in 
71 insects (Post \& Vincent, 1973; Ishaaya \& Casida, 1974; Post et al., 1974). Diflubenzuron has

72 been widely used to control various agricultural and public health pests such as mosquitoes and

73 fly larvae since the 1970 's. It is extremely toxic to young larvae of many mosquito species

74 (Eisler, 1992; Baruah \& Das, 1996; Ali et al., 1999; Zhang \& Zhu, 2006; Zhu et al., 2007). uncertain as to whether BPUs can directly inhibit insect chitin synthase because different studies

77 have yielded inconsistent results. In cell-free chitin synthesizing systems, for example, the BPUs

78 do not inhibit chitin synthesis (Cohen \& Casida, 1980b; Mayer et al., 1981) or block the chitin

79 biosynthetic pathway between glucose and UDP-GlcNAc in intact larvae (Post et al., 1974). In

80 contrast, Nakagawa et al. (1993) showed that diflubenzuron and polyoxin D clearly inhibited the

81 incorporation of $\left[{ }^{3} \mathrm{H}\right]-N$-acetylglucosamine into chitin in isolated intact integument from newly

82 molted American cockroaches. Horst (1981) reported that diflubenzuron can dramatically inhibit

83 CHS activity (approximately 90\%) in the crude microsomes and membrane fractions prepared

84 from brine shrimp larvae.

CHS activity is often measured by a radioactive assay using $\left[{ }^{14} \mathrm{C}\right]$ UDP $-N$-acetyl-D-

86 glucosamine (GlcNAc) as a substrate followed by quantization of insoluble ${ }^{14} \mathrm{C}$-labeled chitin

87 after acid precipitation. The observation that wheat germ agglutinin (WGA) specifically binds to

88 chitin polymer at multiple sites led to the development of a nonradioactive, high throughput

89 screening for antimicrobial agents acting on chitin synthases (Lucero et al., 2002). The

90 sensitivity of this nonradioactive assay method was reported to be similar or even slightly higher

91 than that of the radioactive assay. Also, the method was compatible with a variety of assay

92 conditions, performed using low-cost, widely available commercial reagents, and most helpful

93 when multiple determinations of several samples are required. In this study, we characterized the 
94 enzymatic and inhibitory properties of the chitin synthase prepared from the African malaria

95 mosquito (Anopheles gambiae) by using the nonradioactive assay technique.

\section{Materials and methods}

97 Materials

98 Reagents were purchased from various companies, and each is listed with the company

99 name and catalog number in the parentheses as follows: Trypsin (Sigma-Aldrich, St. Louis, MO;

100 T-1426), soybean trypsin inhibitor (STI) (Fluka BioChemika, WA; 13168), chitin (Sigma-

101 Aldrich; C-9752), protease inhibitor cocktail (Sigma-Aldrich; P-8215), wheat germ agglutinin

102 (WGA) (Bector Lab, Inc. Buringame, CA; L-1020), wheat germ agglutinin-conjugated

103 horseradish peroxidase (WGA-HRP) (Sigma-Aldrich; L-3892), $N$-acetylglucosamine (GlcNAc)

104 (Sigma-Aldrich; A-8625), UDP-GlcNAc (Sigma-Aldrich; U-4375), BCA protein assay kit

105 (Sigma-Aldrich; B-9643), high-sensitivity peroxidase substrate mixtures Colorburst Blue

106 (Alercheck, Inc., Springvale, ME; 90101), 96-well microtiter plates (Corning Incorporated,

107 Acton, MA; 3595), diflubenzuron (Chem Service, West Chester, PA; PS-1028AJ), nikkomycin Z

108 and polyoxin D (Calbiochem, San Diego, CA; 481995 and 529313, respectively), and

109 dithiothretol (DTT) (Sigma-Aldrich; D0632-5G).

110 Mosquito rearing

111 A colony of An. gambiae obtained from the Malaria Research and Reference Reagent

112 Resource Center (MR4) (Manassas, VA) was maintained in the Department of Entomology at

113 Kansas State University (Manhattan, KS) since 2007 by using the same methods as described by

114 Zhang and Zhu (2006). 
115 Crude enzyme preparation, protein content assay, and pretreatment of the enzyme

116 Fifty mosquito pupae were homogenized in $1.0 \mathrm{ml}$ of $50 \mathrm{mM}$ Tris-HCI buffer ( $\mathrm{pH} 7.5)$

117 containing $20 \mathrm{mM}$ of DTT and $1 \mathrm{mM}$ of $\mathrm{MgCl}_{2}$ for 60 s by using a glass-pestle homogenizer.

118 Another $0.5 \mathrm{ml}$ same buffer was used to rinse the homogenizer and combined with the

119 homogenate. The combined homogenate was then centrifuged at $500 \times \mathrm{g}$ for $10 \mathrm{~min}$ to remove

120 unbroken cells, nuclei and debris. The supernatant was carefully transferred to a new tube and

121 used as crude enzyme for following analysis. To obtain the $40,000 \times \mathrm{g}$ fractions, the supernatant

122 were centrifuged at $40,000 \times \mathrm{g}$ for $10 \mathrm{~min}$. Then the supernatant was carefully removed and the

123 pellet was resuspended in the same volume of the same buffer. All preparations were conducted

124 on ice or at $4{ }^{\circ} \mathrm{C}$. Protein determination was carried out in microtiter plate using bovine serum

125 albumin as standard by using the BCA method. To pretreat the enzyme, $10 \mu 1$ of trypsin solution

$126(2 \mu \mathrm{g} / \mu \mathrm{l}$ in buffer) was added to $250 \mu 1$ enzyme preparations in a glass tube and incubate for 10

$127 \mathrm{~min}$ at $30^{\circ} \mathrm{C}$ followed by addition of $10 \mu \mathrm{l}$ of STI solution ( $3 \mu \mathrm{g} / \mu \mathrm{l}$ in buffer). Ten $\mu 1$ of buffer

128 instead of $10 \mu 1$ trypsin solution was used as control without trypsin treatment.

129 CHS activity assays

130 The assay was based on Lucero et al. (2002) with some modifications. In brief, $100 \mu$ of

131 WGA solutions ( $50 \mu \mathrm{g} / \mathrm{ml}$ in deionized $\mathrm{H}_{2} \mathrm{O}$ ) were added to each well of the microtiter plate

132 followed by a $16-\mathrm{h}$ incubation at room temperature $\left(23-25^{\circ} \mathrm{C}\right)$. WGA solutions were removed

133 by vigorously shaking of the plate content. To wash the plate, the empty plate was immersed in

134 a basket of tap water followed by empty the water in the wells by shaking. This washing was

135 repeated two more times to remove the unbound WGA completely. After washing, the wells

136 were blocked by adding $300 \mu \mathrm{l}$ of bovine serum albumin (BSA) blocking buffer ( $20 \mathrm{mg} / \mathrm{ml}$ BSA

137 in $50 \mathrm{mM}$ Tris- $\mathrm{HCl}, \mathrm{pH} 7.5$ ) and incubated for $3 \mathrm{~h}$ at room temperature. After incubation, the 
138 blocking solutions were emptied by shaking. Fifty $\mu 1$ of reaction mixture ( $5 \mathrm{mM}$ GlcNAc, $1 \mathrm{mM}$

139 UDP-GlcNAc in $50 \mathrm{mM}$ Tris-HCI buffer, 7.5) were added to the appropriate wells followed by

140 the addition of extraction buffer and pretreated enzyme $(20 \mu 1)$ to a final volume of $100 \mu 1$. For

141 each assay the corresponding boiled enzyme preparations $\left(95^{\circ} \mathrm{C}\right.$ for $\left.10 \mathrm{~min}\right)$ was used to assess

142 the background readings.

143 The plate was covered with a sealer and incubated at $37^{\circ} \mathrm{C}$ for 60 min by shaking at 100

144 rpm using incubator shaker (New Brunswick Scientific; I2400). To stop the reaction, the plate

145 was emptied and washed as described above for five times, followed by the addition of $200 \mu 1$

146 WGA-HRP $(0.5 \mu \mathrm{g} / \mathrm{ml}$, in blocking buffer $)$ and incubation for $15 \mathrm{~min}$ at $30^{\circ} \mathrm{C}$ with gentle

147 shaking at $100 \mathrm{rpm}$. The plate was emptied by vigorous shaking followed by five times washing

148 as described above. Finally, $100 \mu 1$ peroxidase substrate reagents were added to each well and

149 the optical density (OD) at $600 \mathrm{~nm}$ was determined immediately for $3 \mathrm{~min}$. The content of

150 GlcNAc and the chitin synthase activity in the treatments were calculated by using a standard

151 curve. The standard curve was prepared following the same procedure as described by Lucero et

152 al. (2002). The specific enzyme activity was expressed as nmol GlcNAc.mg ${ }^{-1}$. hour ${ }^{-1}$. Each

153 experiment was repeated 3-4 times, each with triplicate determinations.

\section{In vitro and in vivo inhibition assay}

155 For in vitro inhibition assay, diflubenzuron stock solution $(1 \mathrm{mM})$ was prepared in

156 acetone, whereas polyoxin $\mathrm{D}(1 \mathrm{mM})$ and nikkomycin $\mathrm{Z}(1 \mathrm{mM})$ were prepared in the solvent of

157 acetone: water (1:1). Before use, diflubenzuron was further diluted to $25,5,1$ and $0.2 \mu \mathrm{M}$ by

158 using acetone, whereas polyoxin $\mathrm{D}$ and Nikkomycin $\mathrm{Z}$ were diluted to 25,5 , and $1 \mu \mathrm{M}$ by using

159 acetone:water $=1: 1$. Five $\mu 1$ of each solution was added to $25 \mu 1$ crude enzyme and $20 \mu 1$

160 extraction buffer. The final concentrations of difubenzuron in the reaction mixtures were 2.5, 
1610.5 , and $0.1,0.02 \mu \mathrm{M}$, whereas polyoxin D and Nikkomycin Z were 2.5, 0.5 and $0.1 \mu \mathrm{M}$. Same

162 volume of its own solvent was used as control. The mixture was incubated with shaking at 100

$163 \mathrm{rpm}$ at $37^{\circ} \mathrm{C}$ for $20 \mathrm{~min}$ and the subsequent procedures were same as the enzyme activity assay.

164 For in vivo assay, a series of dilutions of diflubenruon, nikkomycin Z, and polyoxin D

165 were made using acetone. Twenty $\mu \mathrm{l}$ of each chemical were added to a 500-ml glass beaker

166 containing 15 mosquito pupae of 9-h old in $100 \mathrm{ml}$ distilled water and $1 \mathrm{ml}$ fish food. The final

167 concentrations of diflubenzuron were $25,50,100,250$, and $500 \mu \mathrm{g} / \mathrm{L}$, whereas nikkomycin $\mathrm{Z}$ and

168 polyoxin D were 100 and $500 \mu \mathrm{g} / \mathrm{L}$. Same volume of acetone was used as control. After a 24-h

169 (L:D, 16:8) exposure at $25^{\circ} \mathrm{C}$, the pupal mortality was examined and the surviving pupae were

170 collected for crude enzyme preparation followed by enzyme assays based on the same procedure 171 described above. Each control and treatment was repeated four times.

\section{Statistical analysis for enzyme activity}

173 In each assay, the treatment showing the highest specific activity was used as a reference

174 to calculate relative activities in other treatments. The relative activity in percentage was firstly

175 transformed into arcsine square root before one-way ANOVA. Fisher's least significant

176 difference (LSD) multiple comparisons were then used to separate the means of the transformed

177 relative activity or specific enzyme activity among the treatments.

\section{Results}

179 Chitin synthase activity in mosquito pupae

180 Previous study showed that both two CHS genes, $\mathrm{AgCHS1}$ and AgCHS2, are highly

181 expressed in the pupal stage (Zhang et al., 2010), implying an intensive synthesis of chitin in the

182 pupal stage. Thus, we used mosquito pupae to prepare the enzyme for all assays in this study. To 
183 make a standard curve, homogeneous chitin suspension in acetic acid was used for the assay. A

184 high linear correlation is observed when initial rate was plotted as a function of chitin amounts

185 (Fig. S1). Using this standard curve, we examined CHS activity in the crude enzyme. As shown

186 in Fig. S2, CHS activity linearly increased when low amount of enzyme used and reached a

187 plateau phase as the amount of the enzyme increased further. Thus, specific CHS activity of the

188 crude enzyme could be determined by using the data within the linear phase.

Effects of dithiothreitol (DTT) and $\mathrm{Mg}^{++}$on CHS activity

190 To prevent the enzyme oxidation, we added DTT into the buffer for enzyme extraction.

191 Without using DTT, the crude enzyme turned black within a minute and no chitin synthase

192 activity can be detected by using the oxidized enzyme in the subsequent assay. It is obvious that

193 low concentration is not enough to inhibit the enzyme oxidation completely. However, decreased

194 CHS activity was observed when the concentration of DTT was too high (Fig. 1A). Divalent

195 cations have been reported to stimulate CHS activity in insect and other systems. We found that

196 low concentration of $\mathrm{Mg}^{++}$at 1.0-4.0 mM significantly increased CHS activity, whereas 10.0

$197 \mathrm{mM}$ or higher significantly inhibited CHS activity (Fig. 1B).

198 Effects of UDP-GlcNAc and GlcNAc on CHS activity

199 The addition of $0.5 \mathrm{mM}$ UDP-GlcNAc to the reaction mixture slightly increased the CHS 200 activity, whereas high concentration of UDP-GlcNAc significantly inhibited CHS activity (Fig.

201 1C). Similarly, GlcNAc at low concentration in the reaction mixture enhanced CHS activity but 202 at high concentration ( $>10 \mathrm{mM})$ it inhibited CHS activity (Fig. 1D). 
To determine the optimal $\mathrm{pH}$ and temperature for CHS activity, we examined the CHS

205 activity at different $\mathrm{pH}$ and temperature conditions using buffer Tris-HCI. Optimal $\mathrm{pH}$ condition 206 appeared to be $\mathrm{pH}$ 6.5-7.0 (Fig. 2A), whereas optimal temperature ranged between 37 and $44^{\circ} \mathrm{C}$ 207 (Fig. 2B).

209 To evaluate the effect of proteolysis, we measured CHS activity in the presence of 210 trypsin. CHS activity was increased by about 1.2 - and 1.7 -fold in the $500 \mathrm{xg}$ crude enzyme and 211 the successive 40,000 $\mathrm{g}$ fractions, respectively, as compared to those of controls without the 212 addition of trypsin (Fig. 3).

\section{Effect of chitin synthesis inhibitors on CHS activity}

214 The larvae of An. gambiae were highly susceptible to diflubenzuron. Exposure of the 215 third-instar larvae to diflubenzuron at $50 \mu \mathrm{g} / \mathrm{L}$ resulted in about $60 \%$ mortality in $48 \mathrm{~h}$, whereas 216 the classical CHS inhibitors nikkomycin Z and polyoxin D, well established inhibitors of fungal 217 enzymes, exhibited virtually no mortality at $500 \mu \mathrm{g} / \mathrm{L}$ (Table 1 ). We further examined whether 218 these chitin synthesis inhibitors can inhibit CHS in vitro. Very limited inhibition was observed at 219 high concentrations for diflubenzuron and nikkomycin Z, whereas no inhibition on CHS activity 220 was observed for polyoxin D (Fig. 4A). We further exposed the 9-h pupae to these three 221 chemicals under the sublethal concentrations with the mortality within range $0-4.4 \%$ (data not 222 shown). The surviving mosquito pupae were collected at $24 \mathrm{~h}$ and the crude enzyme were 223 prepared from these pupae. In contrast to the in vitro assay, no in vivo inhibition to CHS activity 224 was observed in any of these treatments (Fig. 4B). 


\section{Discussion}

226 Insects have two chitin synthases encoded by two different genes, including CHS1 (also known 227 as $C H S A$ ) and $C H S 2$ (also known as $C H S B$ ). CHS1 is exclusively expressed in the epidermis

228 underlying the cuticular exoskeleton and related ectodermal cells such as tracheal cells, whereas

229 CHS2 is expressed in midgut epithelial cells and responsible for the synthesis of the PM-

230 associated chitin (Merzendorfer and Zimoch, 2003; Arakane et al., 2005, 2008; Zimoch et al.,

231 2005). Similarly, two CHS genes were also identified in An. gambiae (Zhang et al., 2012). Thus,

232 the measurement of CHS activity using crude enzyme preparations in this study comes from the

233 total activity of the two enzymes. However, as the expression of CHS1 is much higher than that

234 of CHS2 (Zhang et al., 2012), therefore the enzyme activity we obtained in this study mainly

235 represents CHS1 activity.

236 Lucero et al. (2002) reported the first alternative to the radioactive assay for CHS activity

237 used since 1957 (Glaser \& Brown, 1957) and successfully applied the assay for measuring fungal

238 CHS activity. In the current study, we first adapted and applied this method for measuring insect

239 CHS activity. The assay provides us a convenient, rapid, cheap and high throughput method for

240 CHS activity assay. Also, the high sensitivity of the assay allows testing of multiple samples

241 containing low amounts of active enzyme. A comparison between two methods showed that this

242 method is even more sensitive as compared with the conventional radioactive method (Lucero et 243 al., 2002).

244 WGA is a chitin-binding lectin with high affinity and specificity for GlcNAc. However, it

245 has been reported that the specificity of this chitin binding assay is lower than the radioactive

246 assay (Kramer \& Muthukrishnan, 2005). To avoid the effect brought by non-specific binding

247 and the effect by endogenous chitin in the crude enzyme, the same amount of the boiled enzyme 
was used as a validation control in this study. No significant differences were observed in the

249 boiled control as the amount of the enzyme was increased. This result indicated that the effects

250 of the endogenous chitin and the non-specific binding were negligible in our assay. Thus, the

251 activity detected in the crude enzyme from mosquito pupae reflects the catalytic activity other

252 than artifacts brought by non-specific bindings or endogenous chitin (Fig. S2).

253 Both $\mathrm{Mg}^{++}$and UDP-GlcNAc were found to stimulate CHS activity at low concentrations

254 but inhibit the activity at high concentrations (Figs. 1B and 1C). Relative high CHS activity was

255 also observed even if $\mathrm{Mg}^{++}$and UDP-GlcNAc were not added (Figs. 1B and 1C). These results

256 suggest that the endogenous $\mathrm{Mg}^{++}$and substrate UDP-GlcNAc in the crude enzyme preparations

257 could sustain the CHS activity and thus produce chitin to some extent. Nevertheless, proper

258 levels of $\mathrm{Mg}^{++}$and substrate UDP-GlcNAc are required to CHS to catalyze chitin synthesis. As

259 one mechanism of enzyme activity regulation, substrate inhibition has also been found in other

260 enzyme systems (Shafferman et al., 1992).

261 GlcNAc, a molecule that is described as an allosteric activator of fungal CHS activity

262 (Merz et al., 1999) has been reported to inhibit enzyme activity at relatively low concentrations

263 (1 mM) for Manduca sexta (Zimoch et al., 2005) as well as for the stable fly (Mayer et al.,

264 1980). Interestingly, we found that GlcNAc stimulated CHS activity at $2.5 \mathrm{mM}$ but inhibited

265 enzyme activity at higher concentrations (Fig. 1D). The mechanism underling the stimulation by

266 GlcNAc for An. gambiae CHS remains elusive. To date, the allosteric activation for CHS was

267 only reported in fungal CHS but not in insect CHS.

268 High CHS activity was observed at temperatures between 37 and $44^{\circ} \mathrm{C}$ (Fig. 2B). In $M$.

269 sexta, high incorporation of UDP-GlcNAc was observed at about $30^{\circ} \mathrm{C}$, and decreased at about

$27040^{\circ} \mathrm{C}$ (Zimoch et al., 2005). In general, CHS activity depends on the membrane fluidity, and thus 
271 CHS activity decreases as temperature increases to $>30^{\circ} \mathrm{C}$. Obviously, our results are not

272 consistent with what was observed in $M$. sexta CHS, but may suggest insect species-dependent

273 variations with respect to optimal temperature for CHS activity.

274 The addition of trypsin not only enhanced the enzyme activity in the crude enzyme

275 preparations but also in the 40,000xg fractions (Fig. 3). To date, very limited information on

276 CHS regulation is available. As a post-translational regulation, the addition of trypsin to cell-free

277 extracts leads to the stimulation of chitin synthesis in fungal and insect systems (Cabib \& Farkas,

278 1971; Cohen \& Casida, 1980a; Mayer et al.1980; Ward et al., 1991; Zimoch et al., 2005),

279 suggesting that inactive CHS is synthesized as a zymogen. However, the in vivo activation

280 factors of CHS remains to be elusive. In some fungal systems, proteolytic fragments associated

281 with CHS activity have been identified (Kang et al., 1984; Machida \& Saito, 1993; Uchida et al.,

282 1996). In M. sexta, trypsin stimulates chitin synthesis in crude midgut extracts but not in

283 membrane fractions. Trypsin-dependent activation was recovered when the soluble fraction was

284 added to the membrane fractions, suggesting that CHS is not directly affected by trypsin but by

285 an unknown soluble factor (Zimoch et al., 2005). However, it is difficult to reveal such a soluble

286 factor as the components of the supernatant are complicated. Later, a chymotrypsin-like protease

287 (CTLP1) that interacts with the extracellular carboxyl-terminal domain of CHS2 in vitro was

288 identified. Highly conserved trypsin cleavage presented in the CTLP1 amino acid sequence

289 suggests that the CTLP1 precursor is activated by trypsin although direct evidence is still

290 missing. It was further suggested that CTLP1 activated by trypsin could stimulate CHS activity

291 (Broehan et al., 2007). A recent study showed that an active, oligomeric CHS complex can be

292 purified from the midgut of the tobacco hornworm (Maue et al., 2009). By using purified 
293 enzymes, it will be very helpful to study the properties of the CHS and its mechanisms of post294 translational regulation.

295 Our study revealed only slight in vitro inhibition of An. gambiae CHS activity by

296 diflubenzuron and nikkomycin $\mathrm{Z}$ at the highest concentration $(2.5 \mu \mathrm{M})$ examined (Fig. 4A).

297 There was no in vitro inhibition by polyoxin D at any concentration examined. Furthermore, no

298 in vivo inhibition was observed by any of these chemicals at any concentration examined (Fig.

299 4B). Indeed, there has generally been lack of report showing that these chemicals are capable of

300 inhibiting CHS activity in vitro to date. The only report of CHS inhibition by diflubenzuron in

301 insects was observed in American cockroaches, in which the isolated intact integument from

302 newly molted cockroaches was used to examining the incorporation of $\left[{ }^{3} \mathrm{H}\right]-\mathrm{N}-$

303 acetylglucosamine into chitin (Nakagawa et al., 1993). It would be interesting to know whether

304 diflubenzuron can inhibit the incorporation of UDP-GlcNAc into chitin polymers by using the

305 isolated intact integument in An. gambiae. Nevertheless, the inhibition of chitin synthesis by

306 diflubenzuron could be due to its effects on other steps of chitin biosynthetic pathways instead of

307 direct inhibition of CHS. Further studies by using purified CHS may help clarify this issue.

308

309

310

311

312

313

314 


\section{Acknowledgements}

316 We thank Sharon Starkey for technical assistance and insect rearing and Ming-Shun Chen for his

317 helpful comments on an earlier draft of this manuscript. This research was supported in part by

318 Kansas Agricultural Experiment Station and NIH (P20 RR016475) from the INBRE Program of

319 the National Center for Research Resources. This manuscript is contribution No. 12-423-J from

320 the Kansas Agricultural Experiment Station, Kansas State University, Manhattan, Kansas, USA.

321 The Anopheles gambiae voucher specimens (voucher No. 211) are located in the Kansas State

322 University Museum of Entomological and Prairie Arthropod Research, Manhattan, Kansas.

\section{Disclosure}

326 All authors have declared no conflicts of interest, including specific financial interests and 327 relationships and affiliations (other than those affiliations listed in the title page of the 328 manuscript) relevant to the subject of this manuscript. 


\section{References}

330 Ali, A., Chowdhury, M.A., Hossain, M.I., Mahmud-UI-Ameen, Habiba, D.B. and Aslam, A.F. (1999) Laboratory evaluation of selected larvicides and insect growth regulators against field-collected Culex quinquefascialus larvae from urban Dhaka, Bangladesh. Journal of the

Arakane, Y., Muthukrishnan, S., Kramer, K.J., Specht, C.A., Tomoyasu, Y., Lorenzen, M.D.,

335 Kanost, M. and Beeman, R.W. (2005) The Tribolium chitin synthase genes TcCHS1 and TcCHS2 are specialized for synthesis of epidermal cuticle and midgut peritrophic matrix, respectively. Insect Molecular Biology, 14, 453-463.

Arakane, Y., Specht, C.A., Kramer, K.J., Muthukrishnan, S. and Beeman, R.W. (2008) Chitin synthases are required for survival, fecundity and egg hatch in the red flour beetle, Tribolium

Baruah, I. and Das, S.C. (1996) Evaluation of methoprene (Altosid) and diflubenzuron (Dimilin) for control of mosquito breeding in Tezpur (Assam). Indian journal of malariology, 33, 61-

344 Broehan, G., Zimoch, L., Wessels, A., Ertas, B. and Merzendorfer, H. (2007) A chymotrypsin-

345 like serine protease interacts with the chitin synthase from the midgut of the tobacco 346 hornworm. Journal of Experimental Biology, 210, 3636-3643.

347 Cabib, E. and Farkas, V. (1971) The control of morphogenesis: an enzymatic mechanism for the 348 initiation of septum formation in yeast. Proceedings of the National Academy of Sciences of 349 the United States of America, 68, 2052-2056.

350 Cohen, E. (1985) Chitin synthetase activity and inhibition in different insect microsomal $351 \quad$ preparations. EXS 41, 470-472. 
352 Cohen, E. (2001) Chitin synthesis and inhibition: a revisit. Pest Management Science, 57, 946353950.

354 Cohen, E. and Casida, J.E. (1980a) Properties of Tribolium gut chitin synthetase, Pesticide 355 Biochemistry and Physiology, 13, 121-128.

356 Cohen, E.and Casida, J.E. (1980b) Inhibition of Tribolium gut synthetase. Pesticide Biochemistry 357 and Physiology, 13, 129-136.

358 Eisler, R. (1992) Diflubenzuron hazards to fish, wildlife, and invertebrates: A synoptic review. 359 Contaminant Hazard Reviews Report, 25, U.S. Fish and Wildlife Service, Laurel, MD. 360 Glaser, L. and Brown, D.H. (1957) The synthesis of chitin in cell-free extracts. Journal of 361 Biological Chemistry, 228, 729-742.

362 Horst, M.N. (1981) The biosynthesis of crustacean chitin by a microsomal enzyme from larval 363 brine shrimp. Journal of Biological Chemistry, 256, 1412-1419.

364 Hunt, S. (1970) Polysaccharide-protein complexes in invertebrates. Academic Press, New York, $365 \quad$ NY.

366 Ishaaya, I. and Casida, J.E. (1974) Dietary TH-6040 alters cuticle composition and enzyme 367 activity of housefly larval cuticle. Pesticide Biochemistry and Physiology, 4, 484-490.

368 Kang, M.S., Elango, N., Mattia, E., Au-Young, J., Robbins, P.W. and Cabib, E. (1984) Isolation 369 of chitin synthetase from Saccharomyces cerevisiae. Purification of an enzyme by 370 entrapment in the reaction product. Journal of Biological Chemistry, 259, $14966-14972$.

371 Kramer, K.J. and Muthukrishnan, S. (2005) Chitin metabolism in insects. In: Gilbert LI, Iatrou 372 K, Gill, SS. (Eds.), Comprehensive MolecularInsect Science, vol. 4. Elsevier, New York, pp. $373 \quad 111-144$. 
374 Lucero, H.A., Kuranda, M.J. and Bulik, D.A. (2002) A nonradioactive, high throughput assay for 375 chitin synthase activity. Analytical Biochemistry, 305, 97-105.

376 Machida, S. and Saito, M. (1993) Purification and characterization of membrane-bound chitin 377 synthase. Journal of Biological Chemistry, 268, 1702 -1707.

378 Maue, L., Meissner, D. and Merzendorfer, H. (2009) Purification of an active, oligomeric chitin 379 synthase complex from the midgut of the tobacco hornworm. Insect Biochemistry and $380 \quad$ Molecular Biology, 39,654-659.

381 Mayer, R.T., Chen, A.C. and DeLoach, J.R. (1980) Characterization of a chitin synthase from the 382 stable fly, Stomoxys calcitrans (L.). Insect Biochemistry and Molecular Biology, 10, 549383556.

384 Mayer, R.T., Chen, A.C. and DeLoach, J.R. (1981) Chitin biosynthesis inhibiting insect growth 385 regulators do not inhibit chitin synthase. Experientia, 37, 337-338.

386 Merz, R.A., Horsch, M., Nyhlen, L.E. and Rast, D.M. (1999) Biochemistry of chitin synthase. $387 \quad$ Exs 87, 9-37.

388 Merzendorfe, H. (2006) Insect chitin synthases: A review. Journal of Comparative Physiology B, $389 \quad 176,1-15$.

390 Merzendorfer, H. and Zimoch, L. (2003) Chitin metabolism in insects: structure, function and 391 regulation of chitin synthases and chitinases. Journal of Experimental Biology, 206, 43933924412.

393 Nakagawa, Y., Matsumura, F. and Hashino, Y. (1993) Effect of diflubenzuron on incorporation 394 of $[3 \mathrm{H}]-\mathrm{N}$-acetylglucosamine $\left(\left[{ }^{3} \mathrm{H}\right] \mathrm{NAGA}\right)$ into chitin in the intact integument from the 395 newly molted American cockroach Periplaneta americana. Comparative Biochemistry and 396 Physiology C, 106C, 711-715. 
397 Post, L.C., de Jong, B.J. and Vincent, W.R. (1974) 1-(2,6-Disubstituted benzoyl)-3-phenylurea 398 insecticides: inhibitors of chitin synthesis. Pesticide Biochemistry and Physiology, 4, 473399483.

400 Post, L.C. and Vincent, W.R. (1973) A new insecticide inhibits chitin synthesis.

$401 \quad$ Naturwissenschaften, 60, 431-432.

402 Richards, A.G. (1951) The integument of arthropods. University of Minnesota Press, $403 \quad$ Minneapolis, MN.

404 Ruiz-Herrera, J. and San-Blas, G. (2003) Chitin synthesis as target for antifungal drugs. Current 405 Drug Targets - Infectious Disorders, 3, 77-91.

406 Shafferman, A., Velan, B., Ordentlich, A., Kronman, C., Grosfeld, H., Leitner, M., Flashner, Y., 407 Cohen, S., Barak, D. and Ariel, N. (1992) Substrate inhibition of acetylcholinesterase:

408 Residues affecting singal transduction from the surface to the catalytic center. $E M B O$ 409 Journal, 11, 3561-3568.

410 Tellam, R.L., Vuocolo, T., Johnson, S.E., Jarmey, J. and Pearson, R.D. (2000) Insect chitin 411 synthase: cDNA sequence, gene organization and expression. European Journal of 412 Biochemistry, 267, 6025-6042.

413 Uchida, Y., Shimmi, O., Sudoh, M., Arisawa, M. and Yamada-Okabe, H. (1996)

414 Characterization of chitin synthase 2 of Saccharomyces cerevisiae. II: Both full size and 415 processed enzymes are active for chitin synthesis. Journal of Biochemistry, 119, 659 -666.

416 van Eck, W.H. (1979) Mode of action of two benzoylphenyl ureas as inhibitors of chitin 417 synthesis in insects. Insect Biochemistry, 9, 295-300. 
418 Ward, G.B., Mayer, R.T., Feldlaufer, M.F. and Svoboda, J.A. (1991) Gut chitin synthase and 419 sterols from larvae of Diaprepes abbreviatus (Coleoptera, Curculionidae). Archives of Insect 420 Biochemistry and Physiology, 18, 105-117.

421 Zhang, D. and Miller, M.J. (1999) Polyoxins and nikkomycins: progress in synthetic and 422 biological studies. Current Pharmaceutical Design, 5, 73-99.

423 Zhang, J.Z. and Zhu, K.Y. (2006) Characterization of a chitin synthase cDNA and its increased 424 mRNA level associated with decreased chitin synthesis in Anopheles quadrimaculatus 425 exposed to diflubenzuron. Insect Biochemistry and Molecular Biology, 36, 712-725.

426 Zhang, X., Zhang, J.Z. and Zhu, K.Y. (2010) Chitosan/double-stranded RNA nanoparticle427 mediated RNA interference to silence chitin synthase genes through larval feeding in the 428 African malaria mosquito (Anopheles gambiae). Insect Molecular Biology, 19, 683-693. 429 Zhang, X., Zhang, J.Z., Park, Y. and Zhu, K.Y. (2012) Identification and characterization of two 430 chitin synthase genes in African malaria mosquito, Anopheles gambiae. Insect Biochemistry $431 \quad$ and Molecular Biology, 42, 674-682.

432 Zhu, K.Y., Heise, S., Zhang, J.Z., Anderson, T.D. and Starkey, S.R. (2007) Comparative studies 433 on effects of three chitin synthesis inhibitors on common malaria mosquito (Diptera: 434 Culicidae). Journal of Medical Entomology, 44, 1047-1053.

435 Zimoch, L., Hogenkamp, D.G., Kramer, K.J. Muthukrishnan, S. and Merzendorfer, H. (2005) 436 Regulation of chitin synthesis in the larval midgut of Manduca sexta. Insect Biochemistry $437 \quad$ and Molecular Biology, 35, 515-527. 
Table 1 Toxicity of chitin synthesis inhibitors to third-instar mosquito larvae.

440

\begin{tabular}{|c|c|c|c|}
\hline \multicolumn{2}{|l|}{ Treatment } & \multirow{2}{*}{$\begin{array}{l}\% \text { Mortality at } 24 \mathrm{~h} \\
(\text { Mean } \pm \mathrm{SE}) *\end{array}$} & \multirow{2}{*}{$\begin{array}{l}\% \text { Mortality at } 48 \mathrm{~h} \\
(\text { Mean } \pm \mathrm{SE}) *\end{array}$} \\
\hline Chemical & Concentration $(\mu \mathrm{g} / \mathrm{L})$ & & \\
\hline Control & 0 & $0.0 \pm 0.00 \mathrm{~d}$ & $0.0 \pm 0.00 \mathrm{c}$ \\
\hline \multirow[t]{4}{*}{ Diflubenzuron } & 6.25 & $2.5 \pm 1.67 \mathrm{~d}$ & $4.9 \pm 2.25 \mathrm{c}$ \\
\hline & 12.5 & $26.3 \pm 8.29 \mathrm{c}$ & $27.5 \pm 8.66 b$ \\
\hline & 25 & $41.3 \pm 5.95 \mathrm{~b}$ & $47.5 \pm 8.66 \mathrm{a}$ \\
\hline & 50 & $57.5 \pm 2.89 \mathrm{a}$ & $60.0 \pm 2.36 \mathrm{a}$ \\
\hline \multirow[t]{2}{*}{ Nikkomycin Z } & 50 & $0.0 \pm 0.00 \mathrm{~d}$ & $0.0 \pm 0.00 \mathrm{c}$ \\
\hline & 500 & $1.5 \pm 1.70 \mathrm{~d}$ & $1.5 \pm 1.70 \mathrm{c}$ \\
\hline \multirow[t]{2}{*}{ Polyoxin D } & 50 & $0.0 \pm 0.00 \mathrm{~d}$ & $0.0 \pm 0.00 \mathrm{c}$ \\
\hline & 500 & $2.1 \pm 1.41 \mathrm{~d}$ & $2.1 \pm 1.41 \mathrm{c}$ \\
\hline
\end{tabular}

441

$442 *$ Same letters indicate no significant difference within each column based on one-way ANOVA

443 followed by Fisher's LSD $(P \geq 0.05)$. 
460 Fig. 1 The DTT, $\mathrm{Mg}^{++}$, UDP-GlcNAc and GlcNAc dependency of CHS activity in mosquito 461 pupae. (A) Enzyme activity was dependent on DTT in the enzyme extraction buffer. (B) The

$462 \mathrm{Mg}^{++}$dependent CHS activity. DTT and $\mathrm{Mg}^{++}$were added to the Tris-HCI buffer for crude

463 enzyme preparation. The concentrations in this figure represented the concentrations in the

464 extraction buffer. UDP-GlcNAc (C) and GlcNAc (D) were added in the reaction mixture and the

465 concentrations in the figure represented the final concentration in the reaction system. The CHS

466 activity values are given in percent of maximal activity and as an average $( \pm \mathrm{SEM})$ of three

467 independent replicates.

469 Fig. 2 Effects of $\mathrm{pH}(\mathrm{A})$ and temperature (B) on the activity of CHS prepared from mosquito

470 pupae. The CHS activity values are given in percent of maximal activity and as an average

$471 \quad( \pm$ SEM $)$ of three independent replicates.

473 Fig. 3 Proteolytic activation of chitin synthesis in different enzyme preparations from the 474 mosquito pupae. Specific CHS activity was measured in the presence (Trp+) and absence (Trp-) 475 of trypsin in 500xg supernatant and successive 40,000xg pellet which was resuspended in 476 proportional volume of the extraction buffer. Asterisks indicate significant difference based on 477 Fisher's LSD $(P \geq 0.05)$.

479 Fig. 4 Comparisons of chitin synthase activity in the crude enzyme preparations following 480 incubation with various concentrations of three chitin synthesis inhibitors (A) and the crude 
481 enzyme preparations from the pupae exposed to the three chitin synthesis inhibitors (B). DF:

482 diflubenzuron; PD: polyoxin D; NZ: nikkomycin Z. Same letters on the error bars indicate no 483 significant difference based on Fisher's LSD $(P \geq 0.05)$.

484

485

486

487

488

489

490

491

492

493

494

495

496

497

498

499

500

501

502

503

504

505

506

507

508

509

510 


\section{$511 \quad$ Fig. 1}

512

513

A

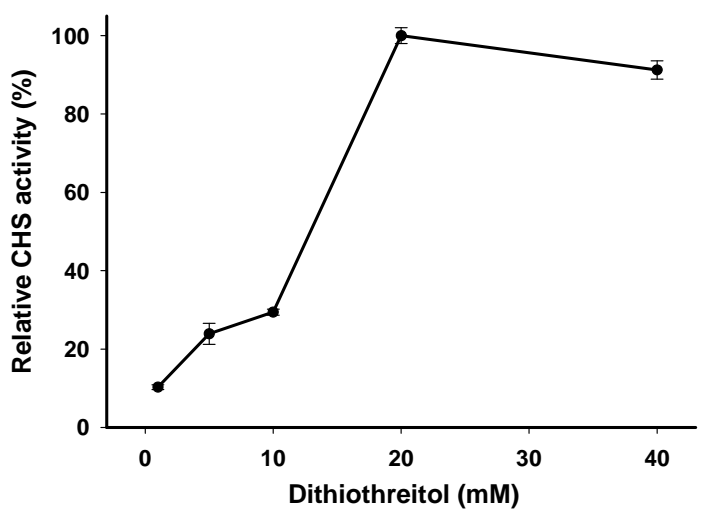

C

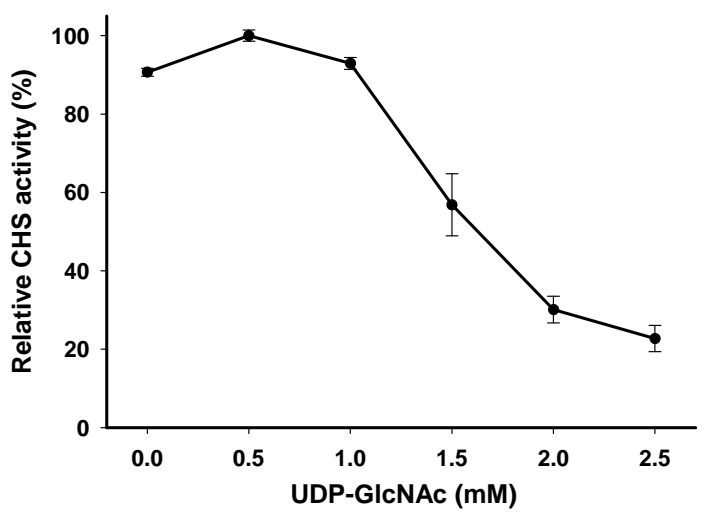

B

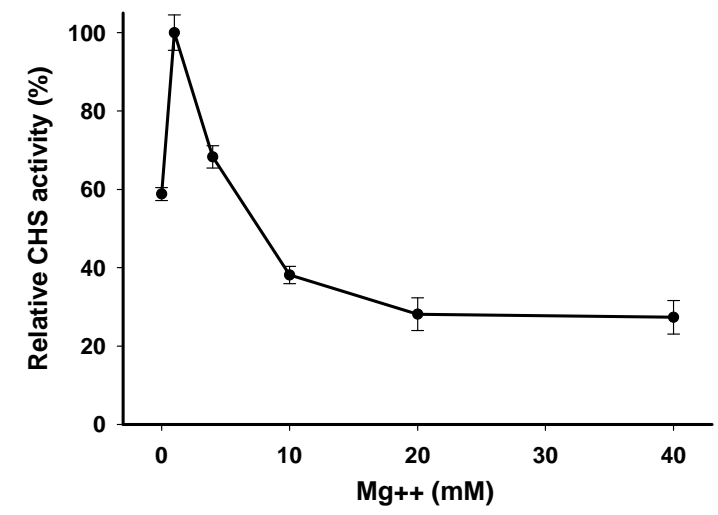

D

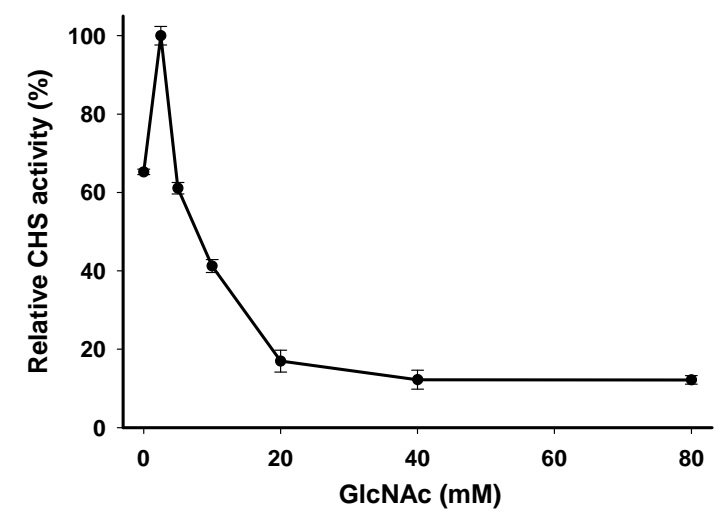


$523 \quad$ Fig. 2

524

A

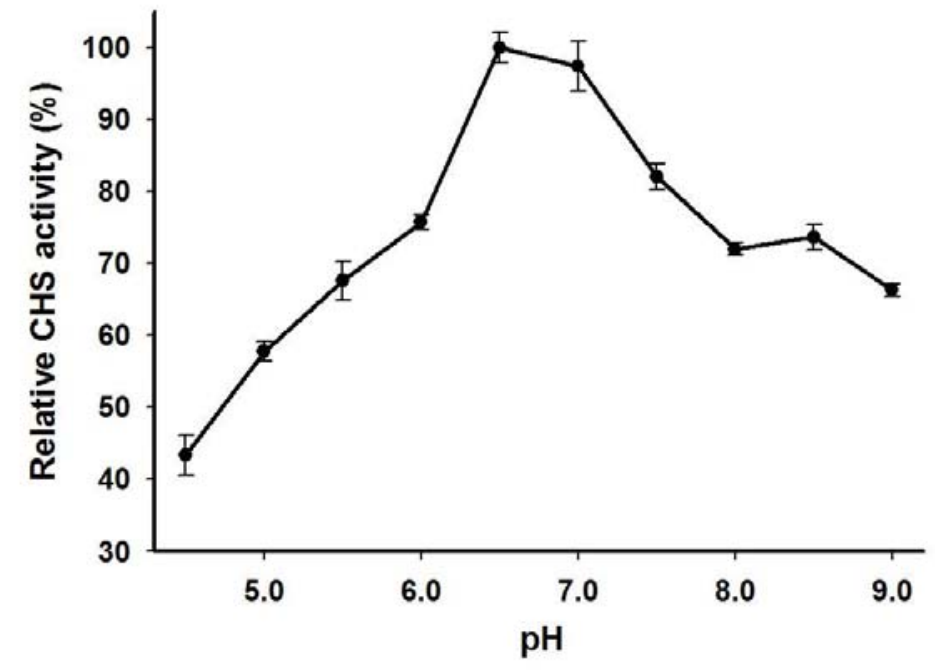

B

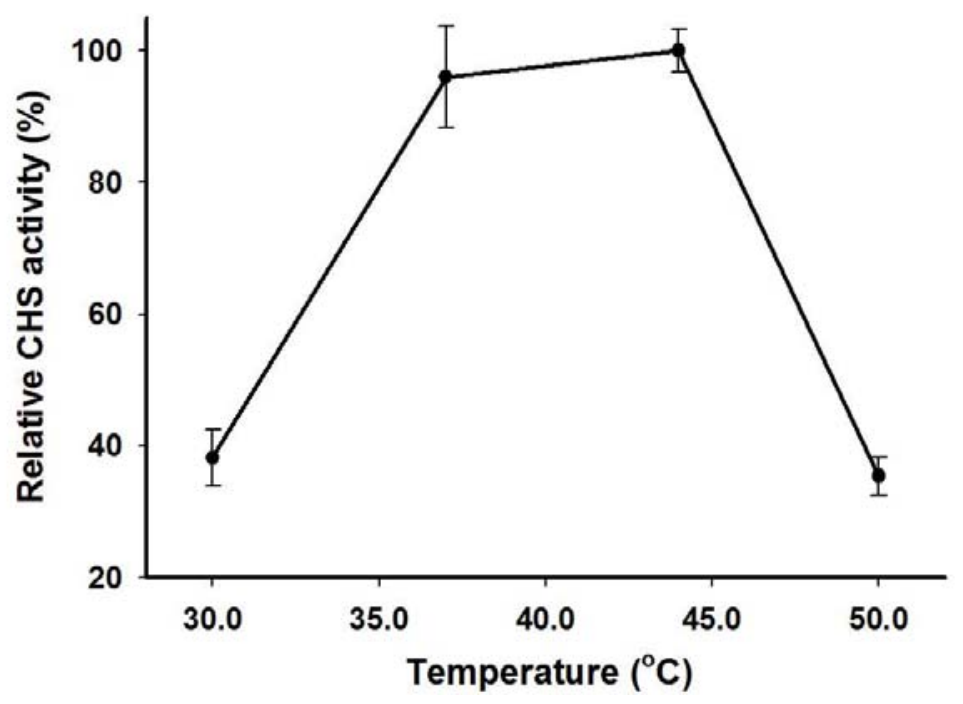

526

527

528 
$531 \quad$ Fig. 3

532

533

534

535

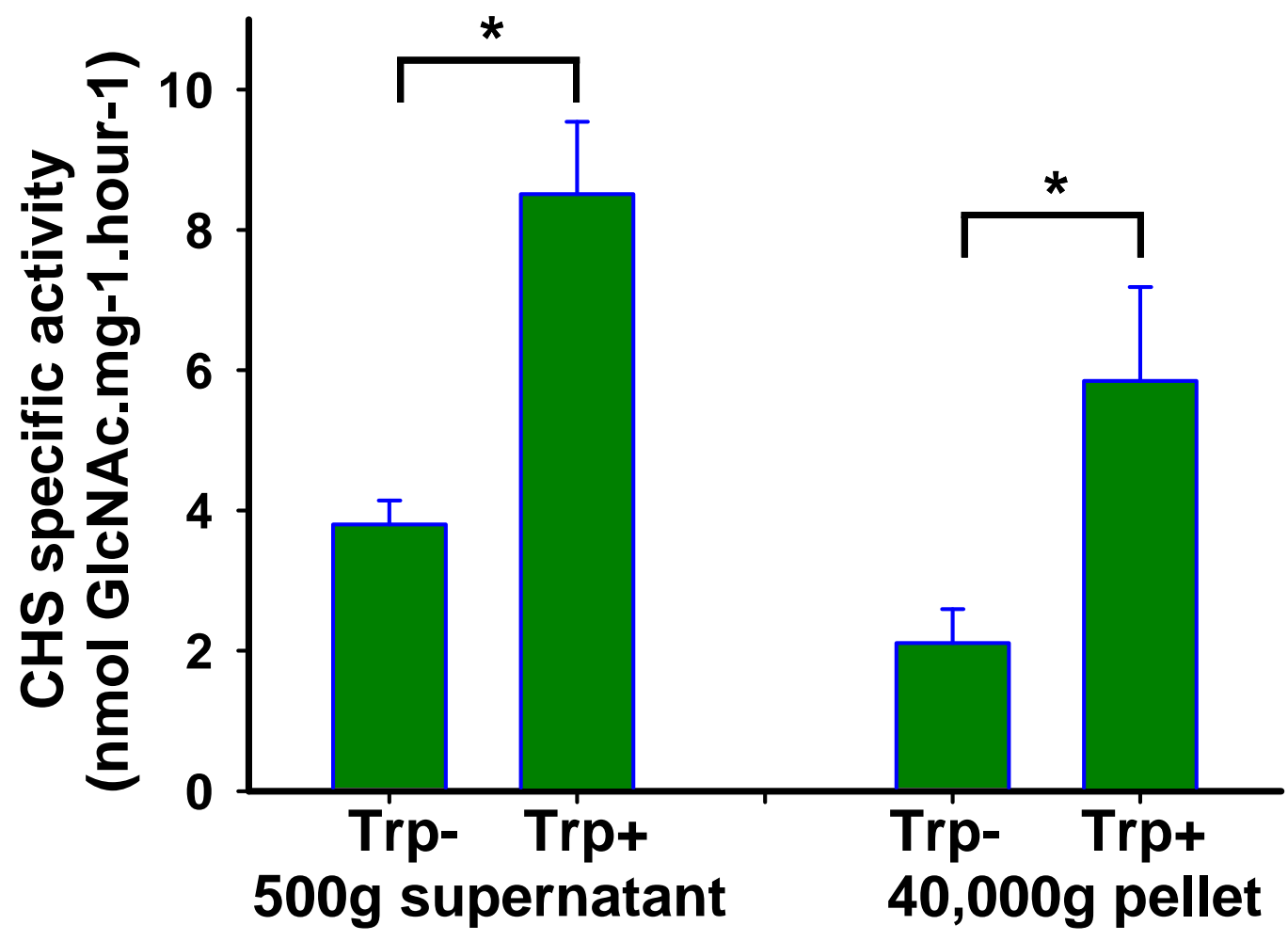


Fig. 4

545

A

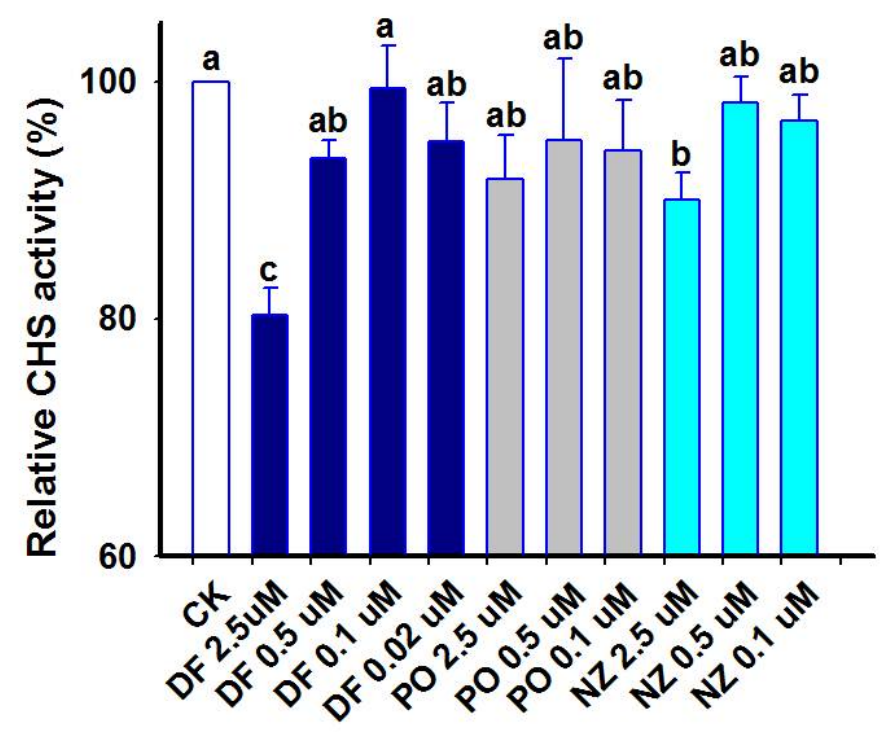

B

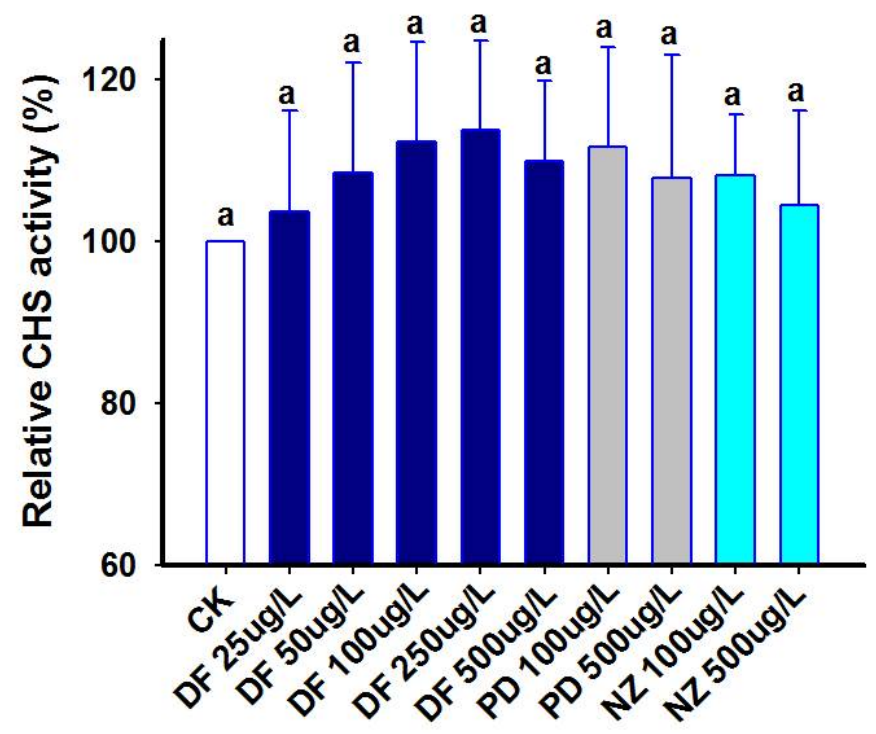

